\title{
El pensamiento crítico y su evaluación en la educación universitaria
}

\author{
O pensamento crítico e sua avaliação na educação universitária \\ Critical thinking and its evaluation in higher education
}

Recibido: 10/03/2021 | Revisado: 15/03/2021 | Acepto: 18/03/2021 | Publicado: 24/03/2021

\author{
María Piedad Rivadeneira Barreiro \\ ORCID: https://orcid.org/0000-0002-5725-6248 \\ Universidad Técnica de Manabí, Ecuador \\ E-mail: maria.rivadeneira@utm.edu.ec \\ Boris Isaac Hernández Velásquez \\ ORCID: https://orcid.org/0000-0002-1057-6877 \\ Universidad Técnica de Manabí, Ecuador \\ E-mail: boris.hernandez@utm.edu.ec \\ Ignacio Loor Colamarco \\ ORCID: https://orcid.org/0000-0003-4806-1032 \\ Universidad San Gregorio de Portoviejo, Ecuador \\ E-mail: iwloor@ sangregorio.edu.ec \\ Karina Luzdelia Mendoza Bravo \\ ORCID: https://orcid.org/0000-0002-0019-3020 \\ Universidad Técnica de Manabí, Ecuador \\ E-mail: karina.mendoza@utm.edu.ec \\ Julio Rivadeneira Barreiro \\ ORCID: https://orcid.org/0000-0002-9494-7494 \\ Universidad Técnica de Manabí, Ecuador \\ E-mail: julio.rivadeneira@utm.edu.ec \\ Lucía Rivadeneira \\ ORCID: https://orcid.org/0000-0001-5989-7703 \\ University of Manchester, UK \\ E-mail: lucia.rivadeneirabarreiro@manchester.ac.uk
}

\begin{abstract}
Resumen
Este trabajo investigativo pretende identificar de forma analítica la relevancia del pensamiento crítico y su evaluación en la universidad. Para ello se ha considerado apropiado efectuar un acercamiento al análisis de su contenido conceptual. Por un lado, las actitudes o disposiciones; y por otro lado, las capacidades o habilidades, pertinentes todos para su debida manifestación, pero que también parecen conformar un proceso racional y lógico ordenado, de forma sistemática; y, cuya identificación es esencial para formular modelos de evaluación debidamente sustentados, que permitan o contribuyan al desarrollo pleno del pensamiento crítico en los estudiantes universitarios y su aplicación para sus actividades profesionales y académicas, proceso que se pretende reforzar hasta el corolario de su práctica, esto es, la denominada "autorregulación". En la introducción se intenta defender la necesidad esencial de promover y desarrollar el pensamiento crítico en los estudiantes universitarios, sea porque se hayan percibido falencias en su ejercicio cotidiano o porque en definitiva constituiría un elemento central de la actividad académica. Posteriormente, se abordan las nociones generales del pensamiento crítico, desglosando sus elementos conceptuales agrupados en dos dimensiones: las disposiciones y las habilidades o competencias, cuyo contenido se repasa. Luego, se discute sobre la evaluación del pensamiento crítico cuya práctica podría contribuir a reforzar esta actividad racional esencial en el contexto estudiantil. Finalmente, en una última sección sobre los retos actuales de la universidad, se plantea la relevancia de la autorregulación que constituye un punto culminante de este proceso racional.
\end{abstract}

Palabras clave: Pensamiento crítico; Autorregulación del aprendizaje; Evaluación; Universidad.

\section{Resumo}

Este estudo visa identificar, de forma analítica, a relevância do pensamento crítico e da sua avaliação no ensino superior. Para tanto, consideramos uma abordagem para a análise do conteúdo conceitual, que abrange dois aspectos: atitudes ou disposições e capacidades ou habilidades. Este último parece conformar, de forma sistemática, um processo racional e lógico ordenado. Esta identificação é essencial para poder apoiar adequadamente modelos de avaliação que permitam ou contribuam para o desenvolvimento do pensamento crítico nos estudantes do ensino superior, essencial para a sua atividade profissional e acadêmica. Este processo é denominado "autorregulação". Na introdução, procura-se defender a necessidade imprescindível de promover e desenvolver o pensamento crítico nos alunos do ensino superior, pois constituiria um elemento vital da atividade acadêmica. Na seção seguinte, são abordadas as noções de pensamento crítico, desdobrando seus elementos conceituais agrupados em duas dimensões: disposições e habilidades ou competências. Posteriormente, é abordada a avaliação do pensamento crítico, explicando a atividade racional essencial 
no contexto acadêmico. Por fim, são apresentados os desafios atuais do ensino superior e levantada a relevância da autorregulação, o que constitui um ponto culminante deste processo racional.

Palavras-chave: Pensamento crítico; Autorregulação da aprendizagem; Avaliação; Universidade.

\begin{abstract}
This study aims to identify, in an analytical way, the relevance of critical thinking and its evaluation in higher education. For this purpose, we have considered an approach to the analysis of the conceptual content, which covers two aspects: attitudes or dispositions, and capacities or abilities. The latter seems to conform, in a systematic way, a rational and logical orderly process. This identification is essential to be able to properly support evaluation models that allow or contribute to the development of critical thinking in higher education students, which is essential for their professional and academic activities. This process is called "self-regulation". In the introduction, an attempt is made to defend the essential need to promote and develop critical thinking in higher education students because it would constitute a vital element of academic activity. In the following section, the notions of critical thinking are addressed, breaking down its conceptual elements grouped into two dimensions: dispositions and skills or competences. Later, the evaluation of critical thinking is covered, explaining the essential rational activity in the academic context. Finally, the current challenges of higher education are presented, and the relevance of self-regulation is raised, which constitutes a culminating point of this rational process.
\end{abstract}

Keywords: Critical thinking; Self-regulation of learning; Evaluation; Higher education.

\title{
1. Introducción
}

Los retos de la Educación demandan que los estudiantes desarrollen un pensamiento crítico (PC) que les permita resolver problemas educativos y sociales; para esto, el papel del docente es importante al crear espacios donde los estudiantes puedan desarrollar estrategias de observación, inferencia, análisis, entre otras.

En este contexto, Poveda (2010), desde la experiencia basada en la observación empírica cotidiana universitaria, ha observado en estudiantes universitarios una presunta tendencia a adoptar fuentes de información sin cuestionarse la credibilidad de sus contenidos, argumentos o fundamentos, ni tampoco la acreditación del autor; aunque sin apoyarse u ofrecer datos empíricos.

Desde otra perspectiva, se suele alegar que la educación tradicional no ha promovido la formación del PC en los estudiantes que llegan a las universidades (Alvarado, 2014).

De todas formas, aunque existiere o no un problema con respecto a la capacidad crítica de los estudiantes de tercer nivel, desde una óptica racional no debería existir discusión en torno a la importancia de fomentar, promover y coadyuvar el desarrollo del PC en los estudiantes universitarios. De allí que, en el presente trabajo, se pretenda abordar la evaluación del pensamiento crítico en la educación universitaria.

\section{Metodología}

Este trabajo tiene como objetivo identificar de forma analítica la relevancia del pensamiento crítico y su evaluación en la universidad. Para este propósito se realizó una revisión teórica de la literatura de acuerdo con lo indicado por Pereira et al. (2018), lo que permite analizar la literatura alrededor del tema estudiado.

A través de bases de datos académicas en línea como Scopus, Google Académico y SCielo se procedió a buscar artículos científicos relevantes al tema estudiado. Se usaron palabras claves como pensamiento crítico, evaluación, universidad, autorregulación y aprendizaje. Se priorizaron estudios que se desarrollaron posterior al 2010, pero se incluyeron algunos artículos previos a ese año por su relevancia en la literatura. Finalmente, se identificaron estudios tanto en lenguaje español, inglés y portugués. Para la selección de los artículos a ser considerados por este trabajo, se analizó el contenido de aproximadamente 50 estudios, de los cuales se procedió a realizar análisis de cada uno de ellos y se seleccionaron aquellos que eran más relevantes para este estudio, mismos que dan soporte a las secciones siguientes. 


\section{Nociones Generales del Pensamiento Crítico}

La necesidad de formar el PC en estudiantes universitarios ha sido mencionada por Rolón (2014), alegando que se puede llevar a cabo a partir de planes de formación y evaluación que los investigadores en el área educativa puedan desarrollar. Para tal efecto es necesario entender de qué se trata el pensamiento crítico, por lo que resulta válido acudir a la definición ofrecida por Elder y Paul (2005, p. 7):

"El pensamiento crítico es el proceso de analizar y evaluar el pensamiento con el propósito de mejorarlo. El pensamiento crítico presupone el conocimiento de las estructuras más básicas del pensamiento (los elementos del pensamiento) y los estándares intelectuales más básicos del pensamiento (estándares intelectuales universales). La clave para desencadenar el lado creativo del pensamiento crítico (la verdadera mejora del pensamiento) está en reestructurar el pensamiento como resultado de analizarlo y evaluarlo de manera efectiva".

De todas formas, la dificultad de definir el PC ha sido mencionada por diversos autores (Facione, 2015; Velásquez de Suárez y Figueroa, 2012); por lo que resultaría más ilustrativo todavía acudir a sus componentes.

En esta línea, el propio Facione (2015), invocando las conclusiones formuladas por un consenso de expertos, ha señalado que el pensamiento crítico estaría compuesto por un conjunto de "habilidades cognitivas y disposiciones", comprendiéndose entre las habilidades cognitivas las siguientes: "interpretación, análisis, evaluación, inferencia, explicación y autorregulación”. En la misma vía Chrobak (2017) postula que el PC se manifestaría como producto de un proceso resultante de la combinación de la actitud, del conocimiento y habilidades de pensamiento.

Pudiendo entenderse, en todo caso, que el tratamiento de los conceptos asociados al PC resultaría no solo más didáctico, sino esencial, si de lo que se trata es de contribuir a su desarrollo y fomento así como a su evaluación. Para tal efecto, a continuación se tratará de explicar someramente los componentes del PC, en el orden y partiendo de lo indicado por Facione (2015).

- Comprensión.- Se entendería por interpretación a la comprensión de cualquier hecho como producto de un razonamiento que incluye, según Paul y Elder 2005, el ejercicio lógico de la inferencia y de la conclusión.

- Interpretación.- Según la noción de Facione (2015) la capacidad de interpretación incluiría no solo la capacidad de comprender, sino también de explicar el significado de lo analizado, no solo tratándose de un texto sino de una amplia gama de fuentes de información tales como eventos, gestos, situaciones, códigos, y otros; es decir, cualquier fuente sobre la que sea posible comprender y explicar lo entendido; pero este autor no incluye a la inferencia y a la conclusión en esta habilidad. En suma, se podría entender como interpretación a la racionalización de las percepciones resultantes del análisis de algún hecho espontáneo o artificial, es decir, el procesamiento lógico y racional de la información que el ser humano pueda extraer de aquello que le rodea.

- Análisis.- De acuerdo con Facione (2015) el análisis consistiría en el examen racional de un hecho (texto, enunciado, fenómeno, o cualquier otro) y de su posible relación lógica con otros, lo cual incluye la comparación de tales fuentes.

- Evaluación. - La evaluación, de acuerdo con lo indicado por Facione (2015), consistiría en la valoración racional de los análisis y conclusiones formuladas por otros con respecto a hechos de diversa índole, como producto de análisis anteriores.

- Inferencia.- En cuanto a la inferencia Facione (2015) refiere que sería la capacidad de establecer los elementos que se consideren relevantes para formular "conjeturas e hipótesis" así como conclusiones (esta última la incluye como una sub habilidad).

- Explicación.- Para Facione (2015) la explicación consistiría en la capacidad de exponer de forma razonada y coherente el producto obtenido del análisis y evaluación de hechos. 
- Autorregulación.- Para el mismo autor, la autorregulación sería un examen lo más objetivo y franco posible con respecto al producto del propio razonamiento, esto es, el examen de los procesos de análisis, evaluación e inferencia que llevaron a determinadas conclusiones, incluyendo los posibles sesgos que existan.

- Disposiciones.- La disposición o actitud hacia el PC según la noción promovida por Facione (2015) consistiría en una inclinación o interés hacia la resolución de problemas, preguntas o dudas, a través de herramientas racionales para encontrar respuestas veraces. Similar perspectiva es mencionada por Moreno-Pinado y Tejeda (2017).

Como se observa de lo puntualizado en esta parte al desglosar las habilidades cognitivas y la predisposición hacia el pensamiento crítico es posible defender que tales habilidades y actitudes sean esenciales para los estudios universitarios.

\section{Evaluación del Pensamiento Crítico}

El pensamiento es energía que da origen a imágenes que se transforman en palabras para rememorar el mismo. Desarrollar el pensamiento crítico va a depender en cierta manera de estas imágenes, de ejercitar ambos lóbulos cerebrales y de los objetivos que se pretendan alcanzar (Altuve, 2010). Se ha evidenciado en estudios de pensamiento crítico relacionados a la escolarización de que el conocimiento no asegura el desarrollo del pensamiento crítico (Glaser, 1984, Perkins 1985 y Whimbey, 1985, como lo cita López Aymes, 2012). Desde este punto de vista es importante considerar cómo enseñar a los estudiantes a ser críticos, pero además evaluar ese pensamiento.

Existen diversas propuestas de evaluación del pensamiento crítico que se enfocan en diversas dimensiones. Así por ejemplo tenemos a Ennis (2019) que centra su evaluación en clarificación básica de ideas, bases de decisión, inferencias, clarificación avanzada y metacognición. Por otro lado, Paul y Nosich (2019) se enfocan en propósitos, metas, problema a resolver, punto de vista, dimensión empírica y conceptual del razonamiento, supuestos, implicaciones y consecuencias, interpretación e inferencias, dimensiones transversales y afectivas (como lo cita Manassero-Mas y Vásquez-Alonso, 2020).

La evaluación del pensamiento crítico no es fácil de practicar. Se emplean instrumentos de carácter cuantitativo, cualitativo o ambos. Dentro de las herramientas cuantitativas se pueden mencionar algunas tales como Watson-Glaser Critical Thinking Appraisal, Cornell Critical Thinking, The Ennis-Weir Critical Thinking Easy test, Ross Test of Higher cognitive Process, Science Reasoning, etc. Mientras que en las herramientas cualitativas se pueden citar elaboración o análisis de ensayos para evaluar el pensamiento crítico o algunas de sus capacidades, observación en el aula, entrevistas individuales, entre otras (Norris y Ennis, 1989 como lo cita Sierra, Carpintero y Pérez, 2010).

Las pruebas de pensamiento crítico constituyen una herramienta útil para diagnosticar y evaluar los logros esperados de un sistema educativo, pero además sirven para tomar correctivos ante el incumplimiento de metas (Saiz y Fernández, 2012, como lo cita Betancourth-Zambrano, Muñoz-Morán y Rosas-Lagos, 2017). Por tanto, es necesario que los docentes estemos preparados en el manejo de estos instrumentos con el fin de innovar formas de evaluar el pensamiento crítico de nuestros estudiantes.

\section{Los retos de la Educación Contemporánea}

Si bien el proceso de enseñanza aprendizaje ha evolucionado, especialmente fomentando el trabajo colaborativo tan necesario para vincular lo aprendido en la universidad con el campo laboral, aún se debe trabajar para que existan condiciones pedagógicas que fomenten el pensamiento crítico y su evaluación. Así, la educación contemporánea enfrentaría el reto de corresponder ese aprendizaje en el aula con un mundo laboral que emerge como competencia, donde se les ofrece la oportunidad de demostrar sus aptitudes y habilidades para resolver problemas.

De allí que sea necesario desarrollar y evaluar el pensamiento crítico comprendiendo nociones de este como ya han sido mencionadas en líneas anteriores. Además de haber citado a varios autores que exponen sobre el tema, es necesario 
mencionar a Romano (1995) quien menciona 3 categorías dentro del pensamiento crítico: el nivel que se enfoca en inferir, clasificar, comparar, predecir, entre otras; el nivel que se enfoca en resolución de problemas, pensamiento crítico, pensamiento creativo; y el nivel metacognitivo que autorregula los niveles anteriores (como lo cita Andreu-Andrés y García-Casas, 2014).

Desde esta perspectiva, la autorregulación del aprendizaje involucra que los estudiantes estén conscientes de la información que están procesando, disciernan entre argumentos válidos y falacias, desarrollen justamente el pensamiento crítico en donde puedan aplicar estrategias para resolver problemas y plantear interrogantes que valoren nuevas ideas. Las experiencias previas son importantes porque van a conectar con el nuevo aprendizaje y desarrollar en nuestro cerebro nuevas conexiones para tomar mejores decisiones que permitan plantear soluciones a problemas antes no resueltos.

Todo esto nos lleva a pensar que la educación debe mirar hacia el cambio, así como lo menciona Vélez Gutiérrez (2013) cuando destaca la posibilidad de que la educación sea transformadora y no sea un patrón de los sistemas educativos tradicionales basados en dogmas de fe. Es necesario que las nuevas escuelas formen personas diferentes para múltiples propósitos (Rivas, 2004). Por ello, es importante el actuar docente en el aula para inculcar en los estudiantes una actitud positiva hacia la ciencia y el conocimiento científico (Tamayo, Zona y Loaiza, 2015).

\section{Conclusiones}

Pese a que el pensamiento crítico es una construcción conceptual compleja, de la que existen diversas definiciones y enfoques, es posible distinguir al menos dos aspectos esenciales en su contenido: actitudes y capacidades. Las actitudes y capacidades para el pensamiento crítico serían, en esencia, producto de complejos procesos racionales, lógicos y cognoscitivos. La disposición o actitud hacia el pensamiento crítico parecería poder ser percibida aun fuera de los entornos académicos, al funcionar a partir del razonamiento.

Las habilidades para el pensamiento crítico, aunque sean agrupadas o clasificadas de diversas maneras por los distintos autores, generalmente se corresponden con la capacidad de analizar de forma objetiva, racional y lógica las ideas o hechos, a fin de deducir respuestas o soluciones racionales. La academia es o debería ser un templo de la racionalidad sustentada por el pensamiento crítico; por lo tanto, dado que de entre sus estudiantes se forman los futuros académicos; el pensamiento crítico debe ser el puntal de su preparación. La autorregulación constituye quizás el aspecto más avanzado del pensamiento crítico: sería la cúspide del razonamiento; por consiguiente, es capital que se fortalezca su práctica constante. Finalmente, los estudios futuros pueden enfocarse en realizar estudios de caso para evaluar los argumentos presentados en este trabajo de investigación en mayor profundidad.

\section{Bibliografía}

Altuve, J. G. (2010). El pensamiento crítico y su inserción en la educación superior. Actualidad Contable Faces, 13(20), 5-18.

Alvarado P. (2014). El desarrollo del pensamiento crítico: una necesidad en la formación de los estudiantes universitarios. Didac, 64 , $10-17$.

Andreu-Andrés, M. y García-Casas, M. (2014). Evaluación del pensamiento crítico en el trabajo en grupo. Revista de Investigación Educativa, 32 (1), $203-222$.

Betancourth-Zambrano, S., Muñoz-Moran, K. T., y Rosas-Lagos, T. J. (2017). Evaluación del pensamiento crítico en estudiantes de educación superior de la región de Atacama-Chile. Prospectiva. Revista de Trabajo Social e intervención social, 199-223.

Chrobak, R. (2017). El aprendizaje significativo para fomentar el pensamiento crítico. Archivos de Ciencias de la Educación, 11(12).

Facione, P. A. (2015). Critical thinking: What it is and why it counts. Insight assessment, (1), 1-23.

López Aymes, G. (2012). Pensamiento crítico en el aula. Docencia e Investigación, (22), 41-60.

Manassero-Mas, M. A. y Vásquez-Alonso, Á. (2020). Evaluación de destrezas de pensamiento crítico: Validación de instrumentos libres de cultura. Tecné, Episteme y Didaxis: TED, (47). 15-32.

Moreno-Pinado, W. E., y Tejeda, M. E. V. (2017). Estrategia didáctica para desarrollar el pensamiento crítico. REICE. Revista Iberoamericana sobre Calidad, Eficacia y Cambio en Educación, 15(2), 53-73. 
Research, Society and Development, v. 10, n. 3, e51910313748, 2021

(CC BY 4.0) | ISSN 2525-3409 | DOI: http://dx.doi.org/10.33448/rsd-v10i3.13748

Patiño, H. (2014). El pensamiento crítico como tarea central de la educación humanista. Revista Didac, (64), 3-9.

Paul, R. y Elder, L. (2005). Estándares de competencia para el pensamiento crítico. Estándares, Principios, Desempeño, Indicadores y Resultados. Con una Rúbrica Maestra en el Pensamiento Crítico. Recuperado el, 20(3), 2015.

Pereira A. S., Shitsuka, D. M., Parreira, F. J., y Shitsuka, R. (2018). Metodologia da pesquisa científica. Universidade Federal de Santa Maria, UAB/NTE/UFSM. https://urlzs.com/qtZpg.

Poveda, I. L. (2010). Formación de pensamiento crítico en estudiantes de primeros semestres de educación superior. Revista Iberoamericana de Educación, 53(3), 1-7.

Rivas, P. (2004). La formación docente, realidad y retos en la sociedad del conocimiento. Educere, 8(24),57-62.

Rolón, N. (2014). Pensamiento crítico y docencia. Breves reflexiones de su aporte y riqueza. Revista Didac, (64), 18-23.

Sierra, J., Carpintero, E., y Pérez, L. (2010). Pensamiento crítico y capacidad intelectual. Faisca: revista de altas capacidades, 15(17), 98-110.

Tamayo, O. E., Zona, R., y Loaiza, Y. E. (2015). El pensamiento crítico en la educación. Algunas categorías centrales en su estudio. Revista Latinoamericana de Estudios Educativos, 11(2), 111-133.

Velásquez de Suárez, M. y Figueroa, H. (2012). Desarrollo del pensamiento crítico en estudiantes de educación superior en El Salvador. Panorama, 6(10), 720 .

Vélez Gutiérrez, C. (2013). Una reflexión interdisciplinar sobre el pensamiento crítico. Revista Latinoamericana de Estudios Educativos, 2, (9), 11-39. Manizales: Universidad de Caldas. 